\title{
Are dinosaurs the rule or the exception? Developing concepts of death and extinction
}

\author{
Devereaux A. Poling ${ }^{a}, *$, E. Margaret Evans ${ }^{\mathrm{b}}$ \\ a Ohio University-Zanesville, Zanesville OH 43701, USA \\ ${ }^{\mathrm{b}}$ University of Michigan, MI, USA
}

Received 1 October 2003; received in revised form 1 March 2004; accepted 1 April 2004

\begin{abstract}
The development of concepts of individual death and species extinction were examined in two studies. Sixty-eight, 4- to 9-year-old children and their parents participated in Study 1. Although preschoolers had some understanding of the concept of death, the ability to explain death and extinction improved over the school-age years. However, all age groups were reluctant to accept that extinction may be inevitable for all species, despite the ease with which they accepted death as inevitable for all living beings. This resistance was especially strong in the case of human extinction. Study 2 extended these findings with three groups of adults possessing different degrees of biological expertise. Unlike medical students and lay-adults, evolutionary biologists endorsed the inevitability of extinction and generalized this belief to humans. The results suggest that for the lay-public, death and extinction concepts elicit existential concerns and that they are embedded in potentially conflicting intuitive theories: metaphysics and biology.

() 2004 Elsevier Inc. All rights reserved.
\end{abstract}

Keywords: Conceptual development; Extinction; Death; Novice-expert; Naive biology

The rate of species extinction is nearly 1000 times greater than it was before the emergence of humans on earth, a source of grave concern to contemporary biologists and environmental scientists (e.g., Wilson, 1999). Paleoanthropologists have claimed that lay people do not worry about their destruction of the environment because they do not believe that extinction is a realistic threat to life, in particular human life (Leakey \& Lewin, 1995). This claim is empirically investigated in two studies that focus on the relationship between concepts of species death and concepts of individual death, utilizing a developmental framework.

\footnotetext{
* Corresponding author. Fax: +1-740-588-1434.

E-mail address: polingd1 @ ohiou.edu (D.A. Poling).
} 
The death of an entire species cannot be understood by the child who does not grasp the finality of an individual's death. Thus, our first goal in these studies was to construct measures of children's understanding of extinction and of death that were comparable. Further, we evaluate whether humans are treated differently from other species. Finally, to examine the role of biological expertise in the developmental pattern, we investigate whether the beliefs of biological experts differ from those of the lay population. The motivation for the first two goals is provided by the extensive literature on children's concepts of death, which we briefly review. To our knowledge no one has investigated children's concepts of extinction, but the historical emergence of such concepts provides some insights into the potential developmental pattern.

\section{Understanding death}

A mature concept of individual death includes, but may not be limited to the following four components: (1) irreversibility (the knowledge that once dead, an entity cannot come back to life); (2) cessation/non-functionality (the knowledge that death results in the cessation of all bodily function); (3) inevitability (the knowledge that death is universal and inevitable for all living beings); and (4) causality (accurate knowledge of causes of death; Lazar \& Torney-Purta, 1991; Smilansky, 1987). Western children acquire a mature understanding of death somewhere between 6 years of age (Bering \& Bjorklund, 2004; Evans, Poling, \& Mull, 2001; Slaughter \& Lyons, 2003) and 10 years of age (e.g., Lazar \& Torney-Purta, 1991).

Developmental theorists interested in naive theories of biology have examined children's understanding of death in the context of their understanding of the living world. Carey (1999) and Slaughter and Lyons (2003), argue, for example, that children cannot possess a biological understanding of death until they have developed coherent concepts of life and bodily function, which are believed to be in place around 6 years of age. Supporting this argument, in a unique intervention study Slaughter and Lyons (2003) found that 4- and 5 -year-old children who were taught about the body and bodily processes performed significantly better on measures of death concepts than comparable groups who had not received the training. The intervention was based on a vitalistic biology (Inagaki \& Hatano, 2002), which provides children with some understanding of internal organs and their functions (e.g., lungs take in air so we can breathe), well before the child has an accurate knowledge of biological mechanisms (Carey, 1999).

Most studies on children's understanding of death have been based on Smilansky's (1987) identification and standardization of the death concepts, described above, which focus on people (e.g., Slaughter \& Lyons, 2003). Yet, there have been contradictory results on people versus other animal death concepts (e.g., Lazar \& Torney-Purta, 1991; Smilansky, 1987); these appear to be a function of children's familiarity with the stimuli and the negative affect or denial associated with the idea that a parent or pet could die, as well as the nature of the measures.

To resolve some of these issues in the current studies, questions about familiar people or pets were not included. Further, in contrast to previous research, these studies include humans who are not only unfamiliar, but who are from localities that are unfamiliar to 
the children. Additionally, by including three human exemplars and three exemplars of non-human species, the human versus (other) animal comparison can be more rigorously tested than in previous work. Forced-choice questions are used to test children's recognition of the major death concepts identified by Smilansky; the questions and stimuli are randomly ordered and the questions are worded so that they elicit both yes and no responses. These changes should ensure that the pattern of results cannot be explained by response biases or question order. Most importantly, the use of forced-choice questions for the death measures made it easier to compose comparable measures to test the extinction concepts. Even with these precautions, though, the wording of the questions differed slightly between measures, potentially influencing participants' response patterns. Thus, in addition, we examined the reasoning underlying participants' forced-choice responses by including open-ended questions covering the extinction and death concepts.

The separation of the forced-choice and open-ended measures has an added conceptual benefit in that it should reveal whether young children recognize death and its immediate ramifications, prior to their ability to explain death as a biological process. We argue that the mere recognition of death does not necessarily require a coherent understanding of life. As an absence of goal-directed movement, death should be recognizable to all species. Animate objects exhibit self-propelled and goal-directed movement (e.g., Opfer, 2002). A previously animate entity, which no longer exhibits voluntary or involuntary movement is unlikely to regain its former state, yet it differs from inanimate objects: it is no longer a possible predator and it could be a source of food. Thus, the recognition of the state of death should be important for survival. The presence of goal-directed movement is reliably associated with life judgments by children 5 years of age and older (Opfer, 2002). We would predict, therefore, that preschoolers should be able to make death judgments based on the absence of goal-directed movement in animate entities, even if they have little knowledge of bodily function.

When making death or extinction judgments the urban lay-public are likely to treat humans differently from non-human species. Ross, Medin, Coley, and Atran (2003) argue that urban children are constrained by their lack of biological knowledge and forced to rely on a default anthropocentric heuristic in which humans and animals are perceived as distinct (Evans, 2001; Poling \& Evans, 2002). Moreover, to urban participants death and extinction are not merely biological processes but are especially likely to evoke existential concerns (Evans et al., 2001). In the current studies, participants' sensitivity to the differences between humans and other species will be evaluated on all measures, including open-ended probes about what happens after living things die. It is expected that participants' metaphysical concerns are most likely to be elicited when confronted with the death (or extinction) of the human.

\section{Understanding extinction}

Extinction refers to the death or dying out of an entire species. It is a natural biological process that results when the birth rate of a species is lower than the death rate over a period of time due to small climactic or resource changes (background extinction) or when an entire species is subjected to catastrophic insult such as drought (mass extinction; Wilson, 1999). 
Extinction is commonly presented to children in the context of dinosaurs, made salient in natural history museums and popular films. However, it is not known whether children, or even urban lay-adults, have a coherent understanding of species death, or whether they simply understand extinction as the reason why dinosaurs no longer inhabit the earth. Nevertheless, an understanding of species death is an important component of biological knowledge as well as of conceptual development, broadly construed. The historical development of extinction concepts should provide an initial developmental framework for understanding how individuals might construct such concepts over time. During the seventeenth and eighteenth centuries the most coherent, and favored explanation for extinction was provided by Lamarck (1809, as cited in Mayr, 1982). This explanation was that species thought to be extinct are in fact, just earlier versions of existing species. That is, living species are species that have adapted to the environment and their ancestors are the allegedly extinct species that paleontologists have discovered in fossil records. However, this explanation cannot account for the gradual dying out of a species over time. It was not until Darwin's theory of evolution that explanations for background extinction became available. Thus, Darwinian theory, especially natural selection, is arguably necessary for developing a comprehensive understanding of extinction.

Darwinian evolution is notoriously difficult for students to comprehend, even when they endorse evolutionary rather than creationist views (e.g., Evans, 2000; Ferrari \& Chi, 1998). Further, lay-adults are less likely to apply evolution to humans than to non-humans, again indicating that humans are often differentiated from the animal world (Evans, 2001). If lay-adults view evolution from a pre-Darwinian perspective then they might also apply this viewpoint to an understanding of extinction. Moreover, investigations of key concepts, such as extinction, that underlie evolutionary theory should also uncover novel cognitive biases that motivate everyday reasoning, but which prevent evolutionary theory from being easily grasped (Evans, 2001).

\section{Study 1}

The goal of the first study was to extend previous work on children's understanding of death (e.g., Smilansky, 1987) by using a rigorous closed-ended interview format, a method not typically employed in this area, as well as open-ended questions. In addition, concepts of individual death were investigated as part of a broader network of knowledge, namely an understanding of species death. It was predicted that the four components of a death concept would be better understood in the context of individual death than species death, at least at the younger ages, and that children would be less likely to generalize an understanding of dinosaur extinction to other species. In addition, we hypothesized that children and adults would reason differently about the death and extinction of non-human and human species.

\subsection{Method}

\subsubsection{Participants}

Sixty-eight children comprising 3 non-overlapping age groups, and 32 parents (30 females, 2 males) participated: 26,4 - to 5-year-olds (13 females, 13 males), 4.0-5.8 years 
of age $(M=4.9$, S.D. $=0.62) ; 19,6$ - to 7-year-olds (6 females, 13 males $), 6.1-7.9$ years of age $(M=7.0$, S.D. $=0.65) ; 23,8$ - to 9-year-olds (10 female, 13 male), 8.2-9.8 years of age $(M=8.9$, S.D. $=0.46)$. Participants were recruited through day care centers and letters and phone calls to parents in Northwest Ohio, whose names were drawn from a database of collected birth records. During the initial phone call, parents verbally completed a survey about the child's experience with death. ${ }^{1}$ Parents gave written consent and children gave verbal assent prior to participation. Interviews were conducted in participants' homes, at the university, and at participating child care programs.

3.1.1.1. Demographics. Both mothers and fathers of children in this study had completed an average of two years of college. Analyses of variance on mother's and father's educational level by child age-group revealed no significant differences across age groups. Additionally, ANOVAS on parental report of children's learning capacity revealed no age-group differences.

\subsubsection{Materials}

Fourteen photographs were used for the closed-ended questions. Two photographs were used during the warm-up task and the remaining 12 photographs were grouped into 2 sets of 6 . In the first set, each photograph portrayed a single individual in a naturalistic setting (single stimulus): three photographs of humans from different non-western locations and three of familiar animals (zebra, parrot, turtle). The second set of photographs matched the first set in terms of setting and species, but portrayed three human and three animal groups (group stimulus). One photograph of dinosaurs was used during the open-ended questions.

\subsubsection{Procedure}

At the beginning of the interview, each participant was told: "I am going to show you some pictures and ask you some questions about what animals need to stay alive or what happens to them when they die." To establish rapport, participants were presented with photographs of an insect and a chair (X) and were asked: "Can X die?" and "Is X alive?" All children answered at least two questions correctly. No child was excluded from the interview.

\subsubsection{Closed-ended questions}

During the forced-choice interview, each photograph was presented individually, in random order, with the constraint that the human and non-human species were alternated. Participants were asked four randomly ordered individual death questions for each of the six single-stimulus pictures to which they responded yes or no. Each question

\footnotetext{
${ }^{1}$ Seventy-three percent of the children had experienced some type of death in the family (including pets), although only $7 \%$ (4 children) had experienced the death of a person in the immediate family. Seventy-two percent of parents reported talking with their children about God and heaven in relation to death and 60\% reported talking about death related facts (e.g., the burial ritual, the permanence of death). This information was not available for eight children.
} 
tested a different individual death subconcept: (1) Irreversibility: If this X (pictured entity) died, could it become alive again? (2) Non-functionality: If this X died, could it breathe? (3) Inevitability: Could this X live forever? (4) Causality: Does this $X$ need air to stay alive?

Participants were also asked five randomly ordered species death questions for each of the six group-stimuli pictures. Each question tested one species death subconcept: (1) Irreversibility: If all the X's in this world died and they didn't leave any babies, could this kind of X become alive again? (2) Non-functionality: If all the Xs in this world died and didn't leave any babies, could this kind of X breathe? (3) Inevitability I: Will all of this kind of X die someday and not leave any babies, and Inevitability II: Do you think that someday there won't be any of this kind of X on the world at all, because they will all be dead, just like the dinosaurs? (4) Causality: If the air was bad for them, could all of this kind of X die, and not leave any babies? There were two inevitability subconcept questions, one utilizing the dinosaur analogy, since it seemed plausible that a dinosaur extinction question would be easier to understand.

\subsubsection{Open-ended questions}

Next, all participants were asked several open-ended questions about extinction and one about death (see Appendix A). For the children, there was a warm-up task with four questions about the extinction of dinosaurs (using a photograph of a dinosaur). The rest of the questions were asked using the same group stimuli photographs. Instead of a verbal interview format, parents completed all closed- and open-ended questions using a written questionnaire (with the same photographs as stimuli). Parents also rated their level of agreement to an additional 17 Likert-scale items including 10 extinction items, which will be reported in the second study.

\subsubsection{Scoring}

The closed-ended death and extinction questions were phrased to elicit yes/no responses ( no $=0$; yes $=1$ ). Scores were compiled across the three exemplars with a range of 0-3 (chance $=1.5$ ) for each subconcept by stimulus set (human, animal) measure. Some items were reverse coded (e.g., irreversibility items) to create measures of death and extinction in which all scores were consistent across subconcepts, with higher scores representing agreement with the subconcept. Responses from the two extinction inevitability questions did not differ significantly, thus they were averaged to create a single measure of extinction inevitability.

\subsection{Results}

First, the individual death questions and the extinction questions were analyzed separately. See Fig. 1 for means and standard errors on the agreement scores for the death subconcepts and Fig. 2 for means, standard errors, and differences from chance $(P<0.05)$ on the extinction subconcept agreement scores. Following this section, separate analyses for each of the subconcepts were conducted, in which responses to the individual death and species death questions were compared. Finally, the results of the open-ended questions were examined. 
4- to 5-Years

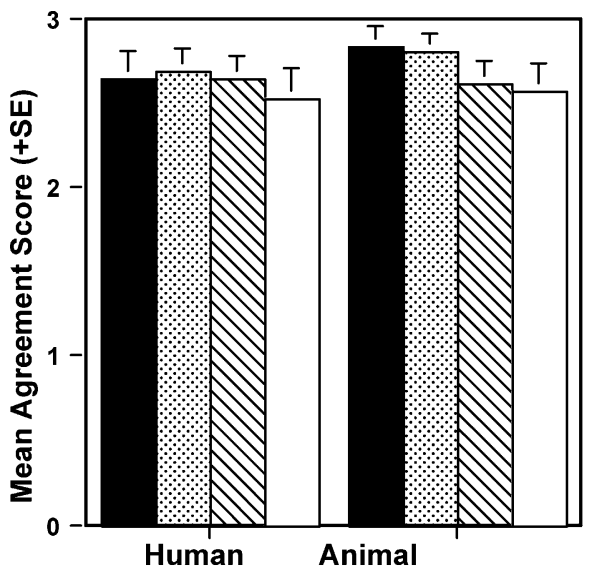

6- to 7-Years

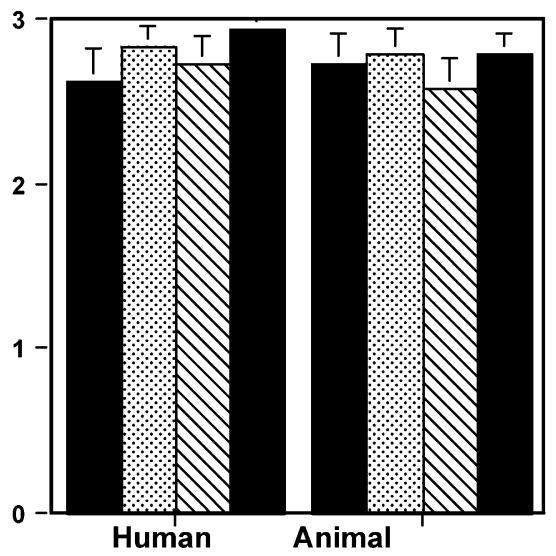

$\nabla$ Inevitabilty

Causality
8- to 9-Years

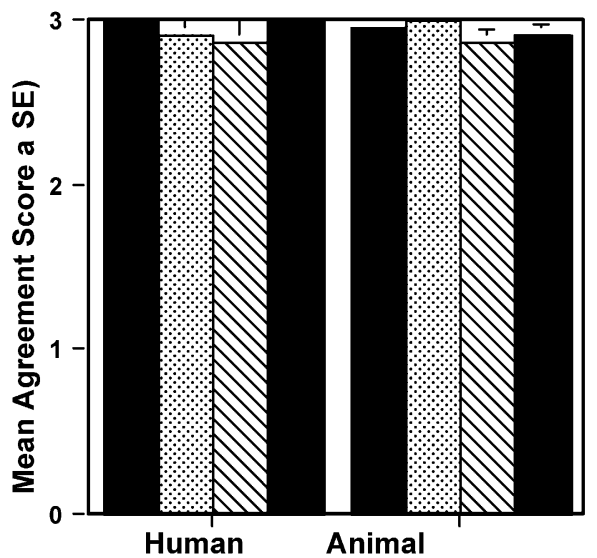

Lay Adults: Parents

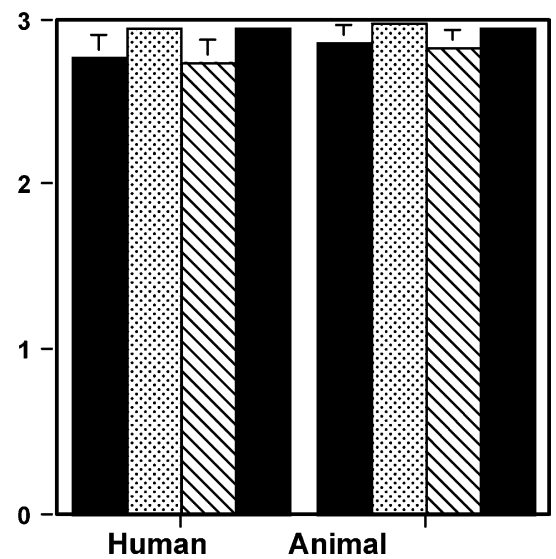

Fig. 1. Children's and parents' scores on four death concepts: irreversibility, non-functionality, inevitability, and causality (forced-choice questions).

\subsubsection{Individual death}

Age group (4), subconcept (irreversibility, non-functionality, inevitability, causality) and stimulus set (humans, animals) were included in a mixed-design multivariate analysis of variance (MANOVA) on participants' responses to the death questions with subconcept and stimulus set as repeated measures. There was a significant main effect for age group, 

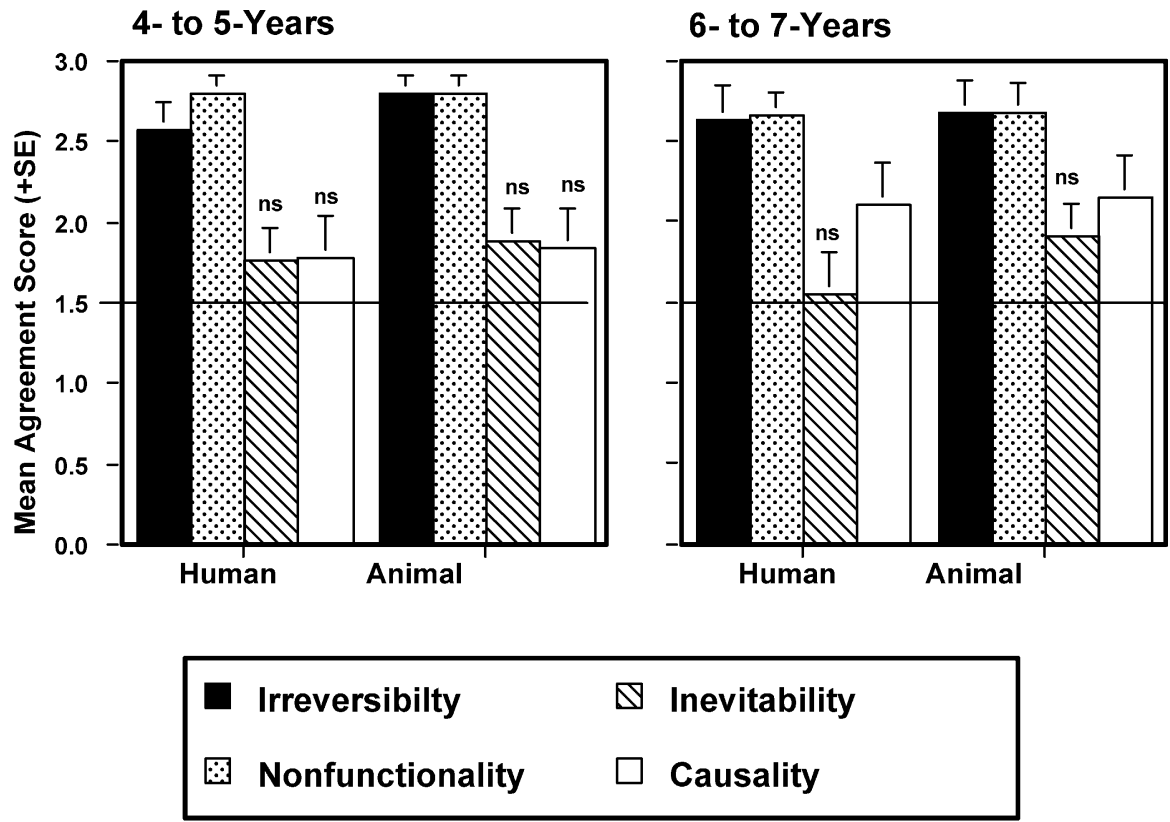

8- to 9-Years

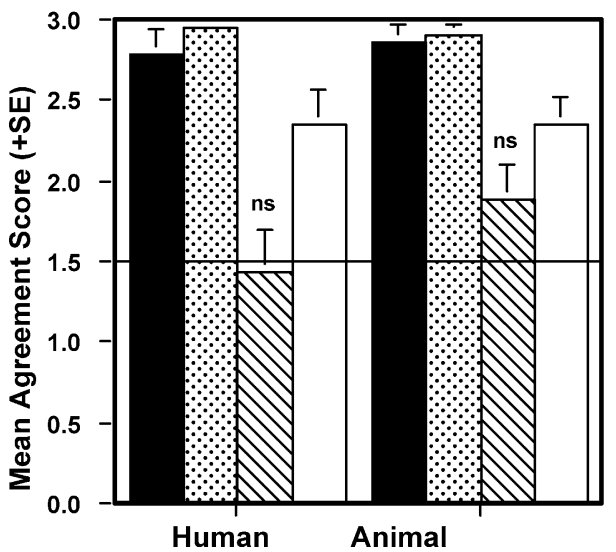

Lay Adults: Parents

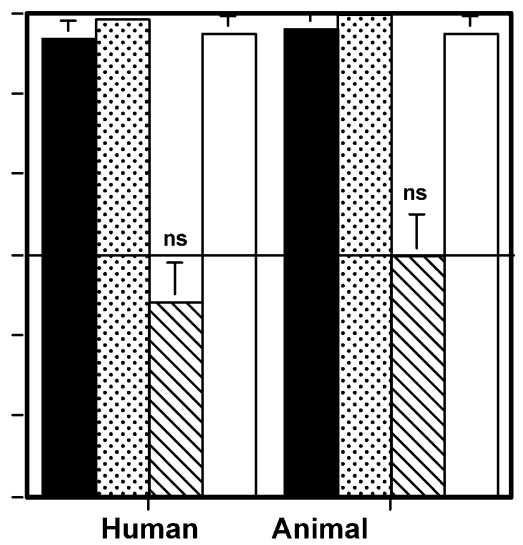

Fig. 2. Children's and parents' scores on four extinction concepts: irreversibility, non-functionality, inevitability, and causality (forced-choice questions).

$F(3,96)=3.12, P<0.05$, only. Bonferroni post hoc comparisons indicated that the 8 - to 9-year-olds had higher scores on the death questions than the 4- to 5-year-olds, $P<0.01$. Preschool children have a relatively good grasp of the four subconcepts of death, which continues to improve with age (see Fig. 1). 
3.2.1.1. Species death. A similar MANOVA, conducted on participants' responses to the extinction questions revealed significant main effects for stimulus set and subconcept, Wilks' Lambda $=0.91$ and $0.44, F \mathrm{~s}=9.79$ and 39.32, and $P \mathrm{~s}<0.005$ and 0.0001 , respectively. There were also significant interactions between stimulus set and subconcept, and subconcept and age, Wilks' Lambdas $=0.90$ and $0.73, F \mathrm{~s}=3.33$ and 3.48 , and $P \mathrm{~s}<0.05$, respectively. Single factor ANOVAs on stimulus set indicated that participants were more likely to view extinction as being inevitable for animals than for humans, $F(1,99)=11.01, P<0.005$ (no other differences were found). In addition, single factor ANOVAs by age group conducted on each of the subconcepts, collapsed across stimulus set, indicated significant effects of age for the non-functionality and causality components, $F$ s $=3.45$ and 7.03 , respectively, $P$ s $<0.05$. Bonferroni post hoc comparisons revealed that the adults were more likely than the 6- to 7-year-olds to believe that extinction implies cessation of function for every species member, $P<0.005$. Adults also had a better understanding of extinction causality than 4- to 5-year-olds and 6- to 7-year-olds, $P$ s $<0.01$.

In conclusion, analyses of the extinction concepts show that the subconcept of inevitability was not endorsed by any age group, with the inevitability of human extinction being particularly problematic. Further, there was an age-related improvement in an understanding of extinction causality and non-functionality (see Fig. 2).

\subsubsection{The four subconcepts: individual death versus species death by age-group}

Age group and question type (individual death, species death,) were included in a mixed-design ANOVA on responses to each subconcept with question type as the repeated measure. There were no significant differences for the irreversibility and non-functionality subconcepts.

3.2.2.1. Inevitability. For inevitability, there was a significant effect of question type, $F(1,96)=85.99, P<0.0001$, with this subconcept endorsed more often for individual than for species death.

3.2.2.2. Causality. The same analysis on responses to the causality subconcept, revealed main effects for age $F(3,96)=7.34, P<0.001$ and question type, $F(1,96)=46.23$, $P<0.0001$, and an age group by question type interaction, $F(3,96)=4.66, P<0.005$. Single factor ANOVAs indicated that all child age-groups understood causality better for individual death than for species death, but there was no difference for adults, $P$ s $<0.005$. In conclusion, children were more likely to understand the causes of individual death than of species death, whereas all participants agreed that individual death is more likely to be inevitable than is species death.

\subsubsection{Open-ended questions}

Two trained independent raters, blind to the age of the participant, coded all open-ended responses (see Appendix A for questions). Cohen's kappa was used to estimate interrater reliability. All discrepancies between the two coders were resolved by the first author. To control for the verbosity of older participants, when more than one answer was provided for a question, only the first answer was coded. In practice, however, fewer than $5 \%$ of the child participants offered more than one answer on any question and their first answer was 
the most complete. Also as answers for the three non-human species were similar, only the first answer was coded.

3.2.3.1. Dinosaur extinction. Cohen's kappa $=0.88$. Children, only, were asked if they knew that the dinosaurs had all died out and were then asked why it had happened. Twelve percent of the 4- to 5-year olds did not know that dinosaurs were extinct and of the remaining preschoolers, most did not articulate a coherent explanation for dinosaur extinction $(65 \%$ either said "I don't know" or said something non-causal, "It was 100 years ago, detectives found their bones"). Those preschoolers who did articulate an explanation (23\%) did not consider causes of species extinction to be different than causes for individual death (e.g., dinosaurs died out "because they got too old"). By ages 6 to 7 years, children expressed mixed ideas, relying primarily on catastrophic explanations (32\%) as well as biological cause explanations, such as bad air $(21 \%)$. A catastrophic explanation was the most popular among 8- to 9-year-olds (52\%), namely the asteroid (meteor) theory of dinosaur extinction, with biological causes a close second $(26 \%)$.

3.2.3.2. Human and animal extinction. Children and adults were asked whether humans and other species could possibly become extinct, just like the dinosaurs (Qu. 5. Cohen's kappa: humans $=0.92$, other species: $=0.90)$. Adults were more likely than children to agree, and all age groups were more likely to endorse this possibility for non-human animals than for humans. Whereas the majority of child age-groups denied that humans might become extinct (58-67\%), there was an age-related increase in the belief that non-humans might become extinct (44-77\%). Adults were more likely to state that non-human rather than human species might become extinct ( $84 \%$ versus $69 \%$ ). All adults and those children who endorsed a belief that species could become extinct were asked about the causes of extinction ( $\mathrm{Qu}$. 6. Cohen's kappa: humans $=0.77$, other species $=0.84$; see Table 1 ). The results confirm the closed-ended data demonstrating an age-related improvement in an understanding of the causes of extinction. However, the asteroid theory of dinosaur extinction was rarely generalized to other species.

3.2.3.3. Human and animal death. Qu. 8, Cohen's kappa: humans $=0.91$, other species $=0.89$ (see Table 2). Children and adults were asked what happens to humans and other species after they die. Overall, responses became more focused with age, with younger children more likely to state that nothing happens. For humans, older children were most likely to cite burial practices, and adults, spiritual responses; whereas for non-humans, decomposition responses predominated.

\subsection{Discussion}

In sum, results from this study suggest that neither children nor adults conceptualize individual death in exactly the same way as they do species death, although there is considerable overlap. Perhaps the most intriguing finding is that no age group exceeded chance levels in their endorsement of the inevitability subconcept for human or animal extinction. This result was found on both extinction inevitability questions, notwithstanding the different wording. In contrast, an acceptance of the inevitability of individual death for both humans 
Table 1

Children's and parents' beliefs about the causes of extinction

\begin{tabular}{|c|c|c|c|c|}
\hline & \multicolumn{4}{|c|}{ Age group (percent of group) } \\
\hline & $4-5$ years & $6-7$ years & $8-9$ years & Adult \\
\hline \multicolumn{5}{|l|}{ Response categories: humans } \\
\hline Spontaneous death (e.g., they can't live forever) & 8 & 10 & 0 & 0 \\
\hline Predator/prey (e.g., humans could kill them) & 11 & 0 & 9 & 0 \\
\hline Biological cause (e.g., no food, bad air) & 11 & 37 & 52 & 22 \\
\hline Catastrophe (e.g., meteor, asteroid) & 4 & 11 & 13 & 19 \\
\hline War (e.g., nuclear war) & 0 & 0 & 0 & 37 \\
\hline Other (e.g., human stupidity; God) & 4 & 21 & 4 & 6 \\
\hline No response or don't know & 62 & 21 & 22 & 16 \\
\hline \multicolumn{5}{|l|}{ Response categories: other species } \\
\hline Spontaneous death & 4 & 16 & 0 & 0 \\
\hline Predator/prey & 8 & 5 & 39 & 12 \\
\hline Biological cause & 19 & 16 & 26 & 38 \\
\hline Catastrophe & 0 & 16 & 13 & 19 \\
\hline War & 0 & 0 & 0 & 12 \\
\hline Other & 8 & 16 & 4 & 10 \\
\hline No response or don't know & 61 & 31 & 18 & 9 \\
\hline
\end{tabular}

and other species was practically at ceiling for all age-groups. The open-ended questions confirmed that the adults had particular difficulty accepting the idea that humans could become extinct.

There are several possible reasons for these results, one of which is that participants' existential concerns were elicited by the extinction inevitability questions, and another of which is that their biological knowledge was not sufficiently detailed. Recall that it was only with the advent of Darwinism that the concept of extinction was convincingly explained

Table 2

Children's and parents' beliefs about what happens after death

\begin{tabular}{|c|c|c|c|c|}
\hline & \multicolumn{4}{|c|}{ Age group (percent of group) } \\
\hline & $4-5$ years & $6-7$ years & $8-9$ years & Adult \\
\hline \multicolumn{5}{|l|}{ Response categories: humans } \\
\hline Nothing (e.g., they just lie there) & 31 & 26 & 8 & 3 \\
\hline Decompose (e.g., they rot, they turn to bones) & 0 & 5 & 0 & 16 \\
\hline Burial (e.g., they get buried, people have a funeral) & 4 & 32 & 70 & 19 \\
\hline Spiritual answer (e.g., they go to heaven) & 35 & 26 & 22 & 53 \\
\hline No response or don't know & 30 & 11 & 0 & 9 \\
\hline \multicolumn{5}{|l|}{ Response categories: other species } \\
\hline Nothing & 46 & 53 & 39 & 6 \\
\hline Decompose & 0 & 10 & 26 & 66 \\
\hline Burial & 4 & 5 & 0 & 6 \\
\hline Spiritual answer & 19 & 21 & 18 & 13 \\
\hline No response or don't know & 27 & 11 & 17 & 9 \\
\hline
\end{tabular}


by biologists (Mayr, 1997). Therefore, before addressing the implications of the results from the first study in more detail, the findings from the parent group should be placed in a broader context. In particular how do they compare with adult populations who have a higher level of expertise in biology, namely in evolutionary theory?

\section{Study 2}

The main goal of this study was to investigate concepts of death and extinction in three adult samples, consisting of the lay-adults from Study 1, medical students, and biology experts. Based on the historical evidence, it was hypothesized that increasing levels of expertise in the biological sciences would underlie a shift in thinking about concepts of extinction. Specifically, expert biologists might be more likely than lay-adults to endorse a belief in the inevitability of extinction. A further goal was to expand the findings from Study 1 by using an additional set of extinction questions with scaled responses, giving adults more flexibility in that they could rate their degree of agreement with the questions. If the pattern of lay-adult responses across the subconcepts is replicated with the new scaled questions, this will indicate that their responses to the earlier questions were driven by the content, not the form, of the question.

\subsection{Method}

\subsubsection{Participants}

Participants were the 32 adults from Study 1 (parent group), 15 medical students (medical student group), and 27 expert biologists (expert group). The total sample of 74 adults consisted of 43 females and 31 males, from 18 to 65 years of age. The majority of participants were white $(n=67)$, the remaining were Hispanic $(n=2)$, multi-racial $(n=2)$, or other $(n=1)$.

4.1.1.1. Parent group. The 32 parents from the first study were from metropolitan areas in the American Mid-West; their knowledge of biology in general and extinction in particular was assumed to reflect that of a lay urban population. Parents' occupations were classified as science or engineering $(n=3)$, health care $(n=7)$, education $(n=7)$, homemakers $(n=$ $9)$, service and retail $(n=4)$ and other $(n=2$ : one attorney and one parent coordinator). Only one parent listed a biology-related profession, "naturalist." Their mean education level, as reported in the first study, was two years of college. Parents self-reported being affiliated primarily with Christian religions: Catholic $(n=12)$, Methodist $(n=8)$, Lutheran $(n=4)$, Christian or Protestant $(n=3)$, Unitarian $(n=1)$, Religious Science $(n=1)$ and Orthodox $(n=1)$, none $(n=2)$.

4.1.1.2. Medical student group. This group of 15 students were enrolled in a Mid-West medical school. They presumably possessed more biological knowledge than the average lay person, but with a focus on applied biology rather than evolutionary theory. Their religious affiliations were: Catholic $(n=7)$, Hindu $(n=1)$, Muslim $(n=1)$, Lutheran $(n=1)$, Eclectic $(n=1)$, none $(n=4)$. 
4.1.1.3. Expert biologist group. Participants were classified as expert biologists if they were working toward or had earned a master's degree or higher in biology and were affiliated with the ecology and evolutionary biology concentration in the biological sciences department at a Mid-West research university. Of the 27 participants in this group, 12 had doctoral degrees and a mean of 14.4 years of scholarly experience since earning the degree, 10 had masters degrees and a mean of 3.9 years of further scholarly experience, and 4 had bachelor of science degrees with a mean of 3 further years of study. Their religious affiliations were: Christian $(n=4)$, Catholic $(n=4)$, Methodist $(n=1)$, Presbyterian $(n=1)$, Lutheran $(n=1)$, Southern Baptist $(n=1)$, Jewish $(n=1)$, Eclectic $(n=1)$, Agnostic/Atheist $(n=2)$, none $(n=11)$.

\subsubsection{Procedure and materials}

The procedure was the same as for the adults in Study 1. In addition to the earlier closed-ended and open-ended questions, all adults completed 17 Likert-scale items in which 10 extinction items (see Appendix B) were intermixed with filler items concerning beliefs about species. The extinction items addressed the subconcepts of irreversibility, non-functionality, inevitability, and causality and were rated using a 5-point scale (1 $=$ strongly disagree; $5=$ strongly agree). Mean scores across all items assessing each subconcept will be reported.

\subsection{Results}

First, responses to the closed-ended death and extinction questions are reported, followed by responses to the Likert-scale items. A possible confound of religious affiliation and biological expertise is investigated before responses to the open-ended questions are reported.

\subsubsection{Closed-ended death and extinction questions}

Separate analyses were conducted for the individual death and species death questions (see Fig. 1 for the parent data). (Since there was no effect for stimulus set in an initial overall MANOVA, responses for humans and other animals were combined.) There were no significant differences on the individual death questions, thus these data will not be reported.

4.2.1.1. Species death. A biology background (parent, medical student, expert) by subconcept MANOVA conducted on responses to the extinction questions revealed significant main effects for biology background, $F(2,71)=7.89, P=0.0008$, and subconcept, $F(3,213)=20.77, P<0.0001$, and a biology background by subconcept interaction, $F(6,213)=7.76, P<0.0001$ (see Fig. 2 for parent data). Single factor ANOVAs and Bonferroni post hoc comparisons on responses to each subconcept indicated a significant effect of biology background for non-functionality, $F(2,71)=4.2, P=0.02$. Parents and biology experts (human: $M=3.0$; S.E. $=0$; animal $M=2.9$; S.E. $=0.1$ ) were more likely than the medical students (human: $M=2.6$; S.E. $=0.2$; animal $M=2.6$; S.E. $=0.3$ ) to endorse this subconcept, $P \mathrm{~s}=0.02$. Such a finding may represent medical students' knowledge of techniques used to revive comatose patients. There was also a sig- 


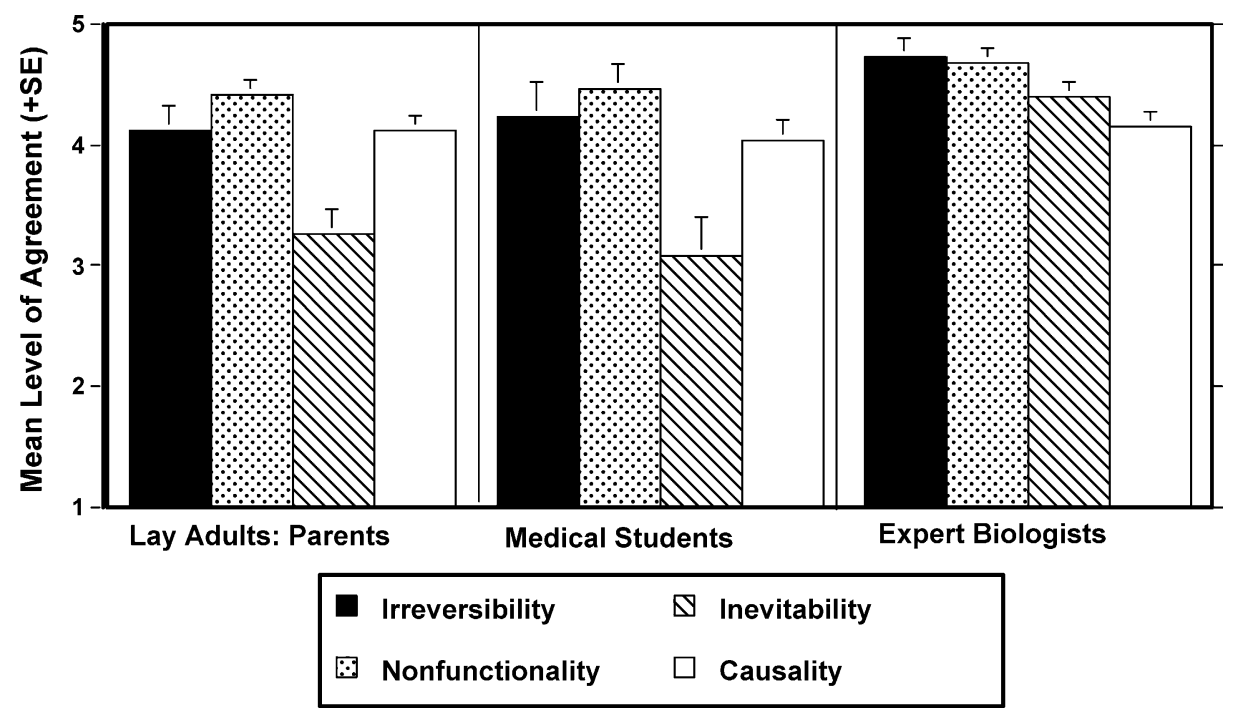

Fig. 3. Parents', medical students', and expert biologists' agreement with four extinction concepts: irreversibility, non-functionality, inevitability, and causality (Likert-scale items).

nificant effect of biology background on inevitability, $F(2,71)=12.19, P<0.0001$, with expert biologists (human: $M=2.7$; S.E. $=0.9$; animal $M=2.9 ;$ S.E. $=0.1$ ) more likely than parents to endorse this subconcept, $P<0.0001$ (medical students: human: $M=2.0$; S.E. $=0.3$; animal $M=2.0 ;$ S.E. $=0.4)$.

\subsubsection{Extinction measure (Likert-scale items)}

Biology background and subconcept were included in a mixed-design MANOVA on participants' responses to the Likert-scale extinction items with subconcept as the repeated measure (see Fig. 3 for means and standard errors). There were significant main effects for biology background and subconcept, $F \mathrm{~s}=10.86$ and $18.00, P \mathrm{~s}<0.0001$, and a biology background by subconcept interaction, $F(6,210)=3.51, P=003$. Single factor ANOVAs revealed that the three groups differed significantly on inevitability, only $F(2,70)=12.62$, $P<0.0001$. Expert biologists were more likely than the other two groups to endorse inevitability $(P \mathrm{~s}<0.0001)$.

\subsubsection{Religious affiliation and biological expertise: a possible confound?}

Although these results show strong support for the hypothesis that biological expertise underlies the acceptance of the inevitability of extinction in adult populations, a possible confound should be addressed. Of the experts, almost half $(n=13)$ did not express a religious affiliation, whereas this was the case for only two of the parents and four of the medical students. To test whether religious affiliation was a factor in these results, a biology background by religious affiliation (yes, no) ANOVA was conducted on responses to the two forced-choice inevitability questions and the Likert-scale inevitability questions. There was a main effect for biology background, only, on all three measures: $F$ s from 5.8 to 11.1; 
$P$ s $<0.005$. A further check for the effect of religious affiliation was conducted within the expert group, as it was the only group in which approximately half of the participants did not express a religious affiliation. In this case, there was a significant effect for religious affiliation on two of the measures: human, forced-choice inevitability (religious affiliation: $M=$ 2.3, S.D. = 1.2; no affiliation: $M=3$, S.D. $=0$ ), and non-human species, forced-choice inevitability (religious affiliation: $M=2.8$, S.D. $=0.4$; no affiliation: $M=3$, S.D. $=0$ ): $F \mathrm{~s}=4.2$ and 5.2; $P \mathrm{~s}=0.05$ and 0.03 . Clearly, this is not a strong effect, but it has to be taken into consideration when evaluating the overall pattern of results.

\subsubsection{Open-ended questions}

Coding of the open-ended questions (see Appendix A) was carried out in the same way as in the first study, though, in contrast to the first study, all responses to each question were coded. Thus, the percentage of responses out of the total $(T)$ for each group will be reported.

4.2.4.1. Species extinction. One hundred percent of the medical students and $96 \%$ of the expert group responded that it is possible that humans could all die out and become extinct, just like the dinosaurs (Qu. 5). For non-human species, 100\% of both the medical student and expert group responded in the positive. As reported earlier, parents were less likely to endorse the idea that species might possibly become extinct someday and this was especially true for humans. Participants were also asked about the causes of species extinction (Cohen's kappa: humans $=0.86$, other species $=0.81$; see Table 3 ). The expert biologists were less likely than the other two groups to mention war and more likely to mention other mass extinction causes (e.g., asteroid). Interestingly, none of the participants referred to geological time in their responses.

Table 3

Parents', medical students', and expert biologists' beliefs about the causes of extinction

\begin{tabular}{|c|c|c|c|}
\hline & \multicolumn{3}{|c|}{ Percent of total responses $(T)$} \\
\hline & Parent $(T=51)$ & Medical student $(T=25)$ & Expert $(T=46)$ \\
\hline \multicolumn{4}{|l|}{ Response categories: humans } \\
\hline Predator/prey & 6 & 8 & 2 \\
\hline Biological cause & 25 & 36 & 37 \\
\hline Catastrophe & 30 & 28 & 42 \\
\hline War & 27 & 24 & 15 \\
\hline Other & 4 & 4 & 4 \\
\hline \multirow[t]{2}{*}{ No response or don't know } & 8 & 0 & 0 \\
\hline & $T=52$ & $T=26$ & $T=52$ \\
\hline \multicolumn{4}{|c|}{ Response categories: other species } \\
\hline Predator/prey & 17 & 27 & 17 \\
\hline Biological cause & 33 & 35 & 38 \\
\hline Catastrophe & 25 & 27 & 31 \\
\hline War & 13 & 8 & 10 \\
\hline Other & 6 & 3 & 4 \\
\hline No response or don't know & 6 & 0 & 0 \\
\hline
\end{tabular}


Table 4

Parents', medical students', and expert biologists' beliefs about what happens after death

\begin{tabular}{lccc}
\hline & \multicolumn{2}{l}{ Percent of total responses $(T)$} & \\
\cline { 2 - 4 } & Parent $(T=37)$ & Medical student $(T=16)$ & Expert $(T=34)$ \\
\hline Response categories: humans & & 6 & 0 \\
$\quad$ Nothing & 3 & 56 & 76 \\
Decompose & 3 & 6 & 18 \\
Burial & 27 & 26 & 3 \\
Spiritual answer & 59 & 0 & 3 \\
Other & 0 & 6 & 0 \\
No response or don't know & 8 & $T=15$ & $T=30$ \\
& $T=35$ & 13 & 0 \\
\cline { 2 - 4 } & & 74 & 31 \\
Response categories: other species & 9 & 0 & 3 \\
Nothing & 66 & 0 & 3 \\
Decompose & 6 & 0 & 0 \\
Burial & 11 & 13 & \\
Spiritual answer & 0 & & \\
Other & 8 & & \\
No response or don't know & & & \\
\hline
\end{tabular}

4.2.4.2. Human and animal death. (Cohen's kappa: humans $=0.74$, other species $=0.69$; see Table 4). In response to the question, What usually happens to Xs after they die?, parents were most likely to provide a spiritual answer, whereas the medical students and the expert group were more likely to provide answers addressing the decomposition of biological matter.

\subsection{Discussion}

These results suggest that biological expertise does support a shift in reasoning about species death. Specifically, expert biologists were more likely to generalize the subconcepts of individual death to species death; in particular, they were more likely to endorse a belief in the inevitability of extinction than the other participants. This was true even though the lay-adults were now given the opportunity to express how much they agreed with the subconcepts, using scaled responses. Additionally, the expert biologists provided open-ended responses that emphasized confidence in their belief in the inevitability of extinction. In response to "Is it possible that all people could die out and become extinct, just like the dinosaurs," one expert biologist wrote, "Of course! We are just another species on the planet." Also, not surprisingly, the expert biologists generally avoided spiritual answers to, "What happens after something dies," in favor of more biologically or ecologically based responses, such as decomposition (see Table 4). For example, one expert biologist wrote, "[After people die]" their organic matter is reintegrated into the soil by the action of decomposers. The "energy" stored in their bodies will then be available for other organisms in the trophic chain. All organisms are made of the same basic structural units, therefore the answers for the [animal] questions are the same". 


\section{General discussion}

"More than 99.9 percent of all evolutionary lines that once existed on earth have become extinct" (Mayr, 1997, p. 198). With this as a baseline, a reasonable prediction would be that contemporary species, including the human, will also become extinct. Experts in evolutionary theory endorsed this prediction. However, children from urban environments, their parents, and medical students were unlikely to accept the idea of the inevitability of species extinction, particularly for the human. What motivates such reasoning? Before commenting in more detail on the implications of these findings for children's and adults' concepts of biology in general and species death in particular, we first comment on what new knowledge these studies contribute to the extensive body of research on children's understanding of individual death. Then we examine the ways in which concepts of individual death are applied at the species level.

\subsection{Death concepts}

By the late preschool years most children in the first study recognized that death is permanent and universal for both humans and animals; in addition, they realized that dead things do not breathe and that death can be caused by lack of air. Even so, there was an age-related improvement over the elementary-school years. Although the current findings are in accord with recent work (Bering \& Bjorklund, 2004; Slaughter \& Lyons, 2003), they contrast with much of the early literature. The earlier research concluded that children do not have mastery of death concepts until about the middle of the elementary-school years and that human death is treated differently from the death of other species (e.g., Lazar \& Torney-Purta, 1991).

The discrepancy between the results from the current research and those from a decade or more ago, could be attributed to several factors, including differing levels of expertise in the children, a related selection effect, and the different methods employed by the researchers. Contemporary children may have more experience with death due to increasingly explicit media. In addition, almost all parents in the first study reported talking to their children about death. Thus, there could be a selection bias; indeed, one mother declined to participate, stating, "We don't talk to our kids about that." It is possible that those children whose parents do not discuss death with them possess a less sophisticated understanding of death and did not participate in this study. Yet, this selection bias should apply to all such studies, even the earlier ones.

More interesting than the effects of a selection bias, we argue that the early competency seen in this study results from the use of novel forced-choice measures, with unambiguous questions requiring yes or no responses. These measures reveal a different kind of competency than those used in the earlier studies that relied in whole or in part on children's explanations. Specifically, young children's ability to recognize death as a unique state, an absence of goal-directed movement, may be separable from an understanding of death that is embedded within a broader conceptual framework. Acknowledging death is a necessary step in developing a sophisticated death concept and this simple first step may be a precursor to more advanced biological knowledge, rather than a result of it. Moreover, recognition of the critical importance of goal-directed movement could well underlie the 
early teleo-vitalistic biology that has been identified in young children's reasoning (Inagaki \& Hatano, 2002).

Nonetheless, a focus on an intuitive biology obscures what was for the majority of the lay-adults in this study an important aspect of death, especially human death, namely its role in an intuitive metaphysics (Harris, 2000). For the human, the most popular responses to questions about what happens after death reflected spiritual beliefs (e.g., they go to heaven) and cultural practices (e.g., the body is buried, or cremated). Interestingly, parents used the spiritual response more than the burial response, whereas the converse was true for the more prosaic 6- to 9-year-olds. Almost half of the preschoolers, though, stated that nothing happens after someone dies. The expert biologists, on the other hand, rarely gave anything other than decomposition responses for humans or non-human species. Biology experts, lay-adults, and children appear to recruit different explanatory frameworks to explain death. In sum, although the recognition of death as a special state, applied to animate entities only, seems to be well within the preschooler's grasp, the ability to explain death gradually improves over the school-age years.

\subsection{Extinction concepts}

Overall, these two studies suggest that two potentially conflicting intuitive theories, a naive metaphysics and a naive biology, play a role in the lay population's reluctance to apply their concepts of individual death to their understanding of species extinction. Further, while expertise in evolutionary science appears to prompt a shift in thinking about extinction specifically, more broadly it may motivate a shift toward thinking about the balance of all living things. Specifically, we argue that the idea of extinction arouses existential concerns, especially in older participants, and especially with respect to human extinction. The fact that lay-adults accept human extinction as a possibility indicates that they possess some knowledge about this topic, but their reluctance to accept extinction as almost inevitable suggests that such ideas may be psychologically overwhelming. As just detailed, lay-adults were the most likely to give spiritual responses to questions about what happens after death. Further, among the expert biologists, those who had a religious affiliation were more likely than those who did not to reject the idea of the inevitability of extinction. Among lay-adults, reasons for rejecting extinction included human ingenuity as well as God's will, signifying that even those who are not religious seek plausible reasons why humans, in particular, will not become extinct.

Why, in contrast to the urban lay adult or child, can the evolutionary biologist accept the inevitability of human and animal extinction? One possible reason could be the uneasy relationship between traditional Western religious beliefs and a post-Darwinian understanding of species origins (Evans, 2000, 2001). Perhaps the rejection of religion combined with the endorsement of Darwinian theory is associated with the acceptance of the inevitability of extinction. However, analyses of the extinction inevitability measure revealed that the effect of biological expertise overwhelmed that of religious affiliation. Whereas religiosity clearly plays a role in such reasoning, we contend that the effect of biological expertise is pivotal.

Central to beliefs about extinction is the concept of species and their relationships. For the evolutionary biologist the human is just one animal among many. In contrast, for the urban participants in these studies the human is accorded a special status, one that elic- 
its existential concerns (see also, Evans, 2001; Poling \& Evans, 2002). Ross et al. (2003) argue that the anthropocentric perspective of urban populations is a consequence of an impoverished natural environment. This would certainly affect concepts of species, species change and ultimately, species death. From the naturalist's perspective, species are temporary spatio-temporal constructs with permeable boundaries, a marked contrast to the common-sense view in which visible species appear to occupy distinct ecological niches and have fixed boundaries (Atran, 1990). Given the current results and the research described earlier, it would be a mistake to assume that even those lay-adults in contemporary industrial societies who endorse evolution have a post-Darwinian view of species. They are more likely to adopt a pre-Darwinian perspective, such as one described in the earlier historical analysis (Mayr, 1982), in which species adapt to novel environmental conditions, but remain, in effect, the same living kind despite the changes. In that case, extinction would be a moot point. Thus, for several reasons, the beliefs of evolutionary biologists are unlikely to yield an account of extinction that is shared by the urban population.

One intriguing line of investigation would compare the ecological reasoning of urban participants, with that of evolutionary biologists and adherents of Eastern religions, both of whom should appreciate the interconnectivity of living kinds, but for different reasons (Barash, 2001; Evans, 2001). In addition, future studies should focus more on the connection between evolutionary beliefs and extinction concepts, explicitly set within a geological time frame.

\subsection{Conclusion}

The urban lay population, unlike evolutionary biologists, apparently considers the extinction of the dinosaurs to be an exception, not a rule: They are unwilling to view extinction as a realistic threat to life on earth. For most children and lay-adults, the human, in particular, is privileged and able to escape the fate of $99.9 \%$ of the species that ever existed. For the lay public, extinction concepts, like death concepts, appear to be embedded in potentially conflicting knowledge structures, one biological, the other metaphysical. Consequently, the concept of species death held by the lay population differs from that of evolutionary biologists, even though all participants had similar concepts of individual death.

Darwinian evolutionary concepts are central to an understanding of modern biological science and related fields. Thus, investigations of the factors preventing students from grasping extinction and other key evolutionary concepts should provide the basis for interventions that will ensure a better informed lay-public. The knowledge gained from these studies is important both for natural historians who seek to inform the public about the implications of extinction, as well as for cognitive psychologists who seek to identify the intuitive cognitive biases that are likely to scaffold the development of ecological and existential reasoning.

\section{Acknowledgments}

An earlier version of this study was presented at the Biennial Meeting of the Society for Research in Child Development, Minneapolis, MD, April 2001. We wish to thank Peg Hull Smith, Steve Christman, Wallace E. Dixon Jr., Jean Funk, and two anonymous reviewers for 
comments on earlier versions of this paper. In particular, we are very grateful to the parents, children, medical students, and evolutionary biologists who participated in this study.

\section{Appendix A. Study one open-ended questions}

Dinosaurs (child interview only)

1. Did you see the film "Dinosaur?" Did you like it? What was the best part?

2. Did you know that the dinosaurs have all died out, that they are extinct?

3. Why do you think that happened? (causality)

4. Do you think the dinosaurs could ever become alive again? (irreversibility)

Other species (Xs: humans, zebras, parrots, turtles) (children and adults)

5. Is it possible that Xs could all die out and become extinct, just like the dinosaurs? (inevitability)

6. What could make that happen? (causality)

7. Do you think the Xs could ever become alive again? (irreversibility)

8. What usually happens to Xs after they die?

\section{Appendix B. Study 2 extinction items}

1. Once extinct, a species can exist again(irreversibility). ${ }^{\mathrm{a}}$

2. If all the members of one species die that species might exist in the world again someday. (irreversibility). ${ }^{\mathrm{a}}$

3. Extinction is caused by some type of catastrophe in the environment (causality).

4. No species can escape from extinction (inevitability).

5. Extinction is probably the final outcome for all species (inevitability).

6. If a catastrophic event caused severe changes in a particular environment, then this could cause species living there to become extinct (causality).

7. After a species becomes extinct, no member of that species can dream (non-functionality).

8. After a species becomes extinct, no member of that species can breathe (non-functionality).

9. If an animal's environment is suddenly changed, the entire species might disappear (causality).

10. All species that exist today are likely to become extinct, someday (inevitability).

${ }^{\mathrm{a}}$ Reverse coded.

\section{References}

Atran, S. (1990). Cognitive foundations of natural history: Towards an anthropology of science. Cambridge: Cambridge University Press.

Barash, D. P. (2001, February 23). Buddhism and the "subversive science." The chronicle of higher education [On-line]. Available at: www.chronicle.com. 
Bering, J. M., \& Bjorklund, D. F. (2004). The natural emergence of reasoning about the afterlife as a developmental regularity. Developmental Psychology, 40, 217-233.

Carey, S. (1999). Sources of conceptual change. In E. K. Scholnick, K. Nelson, S. A. Gelman, \& P. H. Miller (Eds.), Conceptual development: Piaget's legacy (pp. 293-326). Mahwah, NJ: Lawrence Erlbaum.

Evans, E. M. (2000). The emergence of beliefs about the origins of species in school-age children. Merrill-Palmer Quarterly, 46(2), 221-254.

Evans, E. M. (2001). Cognitive and contextual factors in the emergence of diverse belief systems: Creation versus evolution. Cognitive Psychology, 42, 217-266.

Evans, E. M., Poling, D., \& Mull, M. S. (2001, April). Confronting the existential questions: Children's understanding of death, existence and origins. Poster presented at the Biennial meeting of the Society of Research in Child Development, Minneapolis, MN.

Ferrari, M., \& Chi, M. T. H. (1998). The nature of naive explanations of natural selection. International Journal of Science Education, 20, 1231-1265.

Harris, P. L. (2000). On not falling down to earth: Children's metaphysical questions. In K. Rosengren, C. Johnson, $\&$ P. Harris (Eds.), Imagining the impossible: The development of magical, scientific, and religious thinking in children (pp. 157-178). Cambridge, UK: CUP.

Inagaki, K., \& Hatano, G. (2002). Young children's naive thinking about the biological world. New York: Psychology Press.

Lazar, A., \& Torney-Purta, J. (1991). The development of the subconcepts of death in young children: A short term longitudinal study. Child Development, 62, 1321-1333.

Leakey, R., \& Lewin, R. (1995). The sixth extinction: patterns of lives and the future of humankind. New York: Anchor Books.

Mayr, E. (1982). The growth of biological thought: Diversity, evolution, and inheritance. Cambridge, MA: Harvard University Press.

Mayr, E. (1997). This is biology: The science of the living world. Cambridge, MA: Harvard University Press.

Opfer, J. E. (2002). Identifying living and sentient kinds from dynamic information: The case of goal-directed versus aimless autonomous movement in conceptual change. Cognition, 86, 97-122.

Poling, D., \& Evans, E. M. (2002). Why do birds of a feather flock together? Developmental change in the use of multiple explanations: Intention, teleology, essentialism. British Journal of Developmental Psychology, 20, $89-112$.

Ross, N., Medin, D., Coley, J., \& Atran, S. (2003). Cultural and experiential differences in the development of folk biological induction. Cognitive Development, 18, 25-47.

Slaughter, V., \& Lyons, M. (2003). Learning about life and death in early childhood. Cognitive Psychology, 46, $1-30$.

Smilansky, S. (1987). On death: Helping children understand and cope. New York: Peter Lang.

Wilson, E. O. (1999). The diversity of life. New York, Norton. 